\title{
Turkey's Management of COVID-19: Measures and Strategies of Health Policies
}

\section{FAHRETTIN KOCA}

\author{
Minister of Health, Turkey
}

ORCID No: 0000-0002-9124-8866

ABSTRACT Pandemics and related problems have been an important public health issue throughout history and will continue to be so in the future. Soon after its outbreak in Wuhan, China, in December 2019, COVID-19 was declared as a global pandemic which caught many countries unprepared with exceptions. Turkey was one of them. Having worked on plans and preparations for a possible pandemic since 2004 the Turkish Ministry of Health performed well in curbing the initial spread of the virus after its entrance into the country and has played an important role in Turkey's successful fight against COVID-19 since then. Turkey's successful management of the crisis has been marked by its idiosyncratic approach to the crisis and the robustness of its healthcare sytstem which have helped taking COVID-19 pandemic under control and preventing its devastating consequences.

Keywords: COVID-19, Healthcare, Pandemic, Turkey, Cooperation, Humanitarian Aid 
$\mathbf{T}$ hroughout human history, pandemics have been, and will be, an important public health problem. Countries have witnessed various pandemics, along with their dramatic sociological, demographic and economic consequences. Some of the major pandemics include the plague in the $14^{\text {th }}$ century, the cholera and influenza pandemics in 1847-1848, the Spanish flu in 1918, the Asian pandemic in 1957, the Hong Kong flu in 1968, H1N1 influenza, SARS, and MERS in 2009. Finally, now, we are all, globally, going through COVID-19 together.

COVID-19, which is an infectious disease caused by a newly discovered coronavirus, became a global pandemic in about two months after an outbreak in Wuhan, China, in December 2019. The COVID-19 pandemic differs from previous pandemics in terms of its impact and prevalence across countries and sectors. Due to easy transportation around the world and the broad communication network, no pandemic has spread so fast or led to such extensive information-sharing. Turkey immediately started to take stringent measures even before the virus was declared a pandemic by the World Health Organization (WHO) and managed to delay the entry of the disease to the country for more than two months. Taking advantage of this opportunity, the Turkish Ministry of Health was prepared for the pandemic, having made the necessary arrangements in hospitals and strengthened the healthcare infrastructure. Turkey tightened mea- sures upon observation of the first COVID-19 case in the country on March 11, 2020. Under the coordination of the Turkish Presidency, all the involved parties adopted stringent policy measures and implemented them dynamically.

Further, unlike many countries, the pandemic did not take the Turkish health system by surprise. The Ministry of Health had been working on plans and preparations for a possible pandemic since 2004. The "National Preparation Plan for Pandemic Influenza" had been prepared and published in 2019. The National Plan came into force under Presidential Executive Order No. 30744, dated April 13, 2019. The plan provides information and a framework to help all public and private individuals, institutions and organizations recognize the influenza pandemic, be prepared and act in coordination in the most appropriate way in order to fulfill their roles and responsibilities in the event of a pandemic.

Within the scope of this plan, the deputy ministers of all the involved ministries, as well as representatives of the Turkish Red Crescent, the Presidency of Disaster and Emergency Management (AFAD), the Council of Higher Education (YÖK) and respective departments of the Ministry of Health attend the meetings of the National Coordination Committee for Pandemic. Prepared with a multi-stakeholders approach, the $\mathrm{Na}$ tional Preparation Plan formed the basis for Turkey's management of the COVID-19 pandemic. 
Long before the occurrence of COVID-19 cases in the country, Turkey acted proactively; starting with the healthcare sector, it took the required measures as a whole for risk and crisis management early in January 2020. One of the steps taken for scientifically managing the pandemic was the formation of an operation center. The center, consisting of 15 experts, was established under the Ministry on January 6, 2020 in order to follow developments related to coronavirus around the world instantaneously. Subsequently, the Scientific Advisory Board on Coronavirus affiliated with the Ministry was formed on January 10, 2020 with the participation of scientists from the leading Turkish universities. The decisions and advice of the Committee serve as a guide in the successful management of the process.

In the management of the COVID-19 pandemic, policies in accordance with the principles of risk and crisis management were developed by considering the emerging conditions in almost all aspects of life, including healthcare, travel, education, economy, tourism, security, international relations and solidarity, agriculture, production, free circulation, logistics and transportation. Turkey has implemented a wide variety of measures such as keeping selected cases in hospitals, the establishment of pandemic hospitals, the isolation of patients who tested positive, contact tracing, measures for quarantine and restriction, the lockdown of children, teenagers and people 65 years old and over, travel bans and restrictions,

\section{The rapid decision-making process enabled by the Presidential system played an important role in the implementation and follow-up of the decisions taken regarding the pandemic}

online education in schools and universities, and cancelling outdoor activities in public places and financial measures.

Turkey's fight against the COVID-19 pandemic may be evaluated in three stages. The protection period (January and February 2020) involved raising public awareness, providing border security and making preparations for healthcare infrastructure. The period of active fight against the disease in the following three months (March, April, May) started with the adoption of a strategy that concentrated on controlling the spread of infection, and on diagnosis and treatment. The spread of diagnostic laboratories, early diagnosis and treatment, contact tracing and the management of medication and protective materials came to the fore in this period. The next period, called "Controlled Social Life," began in early June and involves the transition to normalization.

Every crisis becomes an opportunity if it is managed well. Sharing experiences gained in the process is crit- 
Turkey has supported more than 80 countries and some international organizations by providing personal protective equipment, making donations and issuing special permissions for import and export to 65 countries ical for turning them into common benefit for humanity. In this regard, I would like to draw your attention to the following lessons we have learned in the process.

\section{Leadership and Governance}

During the period of the COVID-19 pandemic, Turkey set an example for strong leadership and governance. Decisions to control the pandemic at the social level were made under the direction of scientific committees with the preliminary preparation of the Ministry of Health and the will of the Presidential Cabinet through a multi-sectoral approach. The rapid decision-making process enabled by the Presidential system, which was adopted in 2018, played an important role in the implementation and follow-up of the decisions taken regarding the pandemic. Under the leadership of President Recep Tayyip Erdoğan, Turkey's approach to the pandemic was conducted through the coordination of ministries and provincial administrations. From the very beginning of the pandemic, President Erdoğan constantly informed the public through his speeches, and I, as the Minister of Health, provided information at the press conferences.

There are many institutions and stakeholders that directly or indirectly affect healthcare. As the importance of coordination increased in implementing health policies during the pandemic period, the need to develop different mechanisms in terms of leadership and governance became more evident. In this sense, in order to be prepared for pandemic influenza, which may pose a risk for our country and the whole world, it remains critical to involve the National Coordination Committee for Pandemic already actively at work.

In the context of governance, another issue, the importance and priority of which have become better understood in the new period, is to rapidly make and implement central decisions appropriate to the needs and expectations of the population, and in accordance with the governance approach. Particularly, information infrastructure is of vital importance in realizing this need. Clearly, immediate data flow and the use of this data in decision support systems increase the ability to develop proactive policy.

\section{International Solidarity}

The COVID-19 outbreak has tested not only national healthcare sys- 


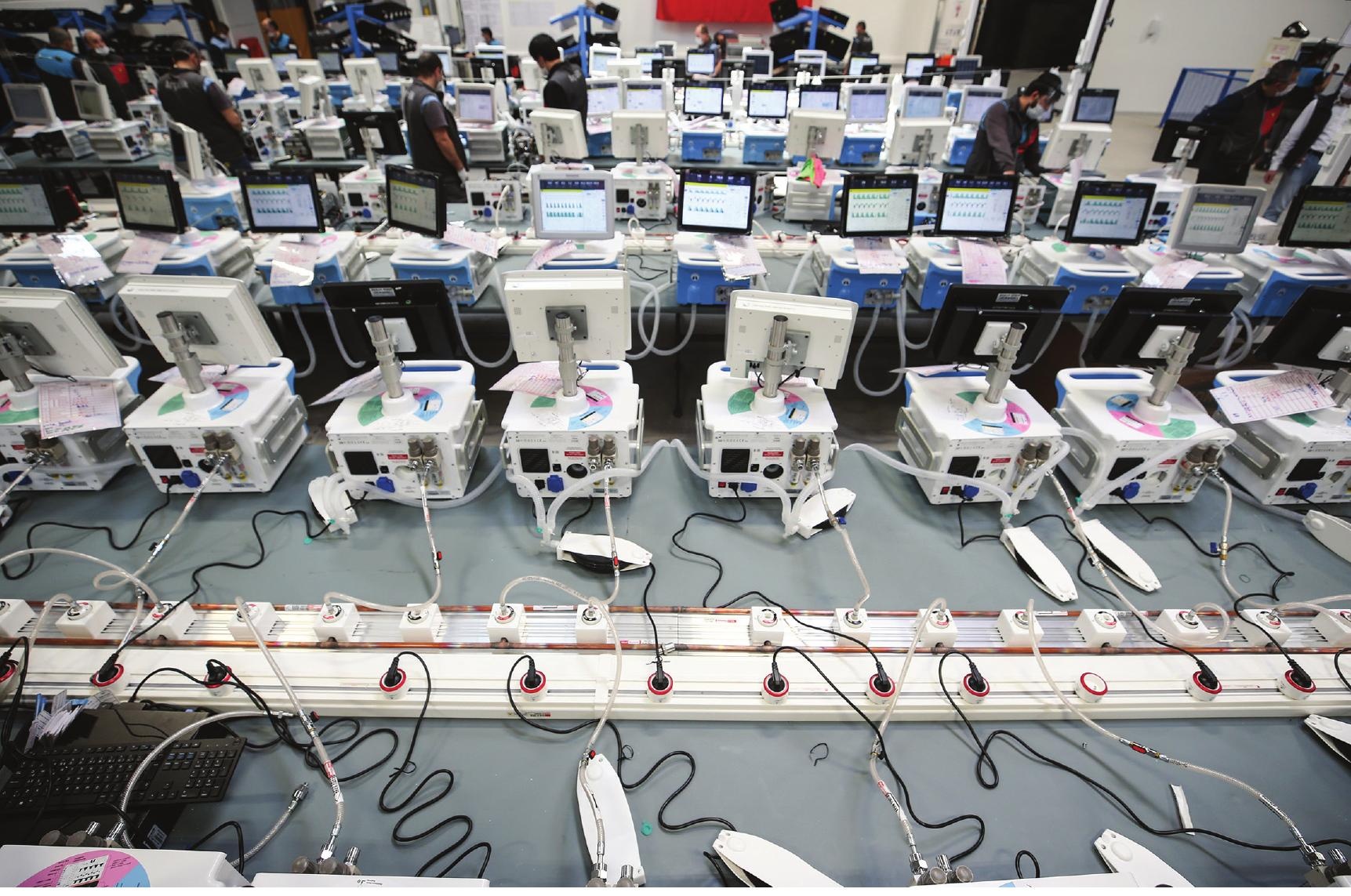


Turkey has supported more than 80 countries and some international organizations by providing personal protective equipment, making donations and issuing special permissions for import and export to 65 countries
Information systems have become the most important tool for the management in early warning, rapid data-based decision making, and the follow-up phase of the process. The strength of healthcare systems depends on qualified human resources and organization as well as user-friendly information technologies infrastructures with certain standards.

\section{Financial Coverage}

Presidency Office of Digital Transformation and the Ministry of Health. Downloaded more than 10 million times since the beginning of June, the application allows person-based tracing in the prevention of the pandemic. The application is constantly updated in order to allow people to follow their families, request face masks and check the risk status of the areas in which they live. After a recent update, it was announced that people planning to travel may obtain a HES code (unique identifier) through this application and travel afterward. So, records of those who travel together will be kept; if a COVID-19 case is detected, the health status of everyone in the party will be checked. Moreover, current applications such as e-Government and e-Pulse are constantly updated. In line with the needs of the population during the pandemic, some features such as the free distribution of face masks, COVID-19 test results, application for pandemic social support, travel permits, etc. have been added to these applications.
Within the scope of Universal Health Insurance (UHI), all individuals, including refugees living in Turkey -whether or not they have social security coverage- are entitled to the benefits of free diagnosis, treatment, follow-up and intensive care treatment associated with COVID-19. Medicines not covered by General Health Insurance, but included in the treatment algorithm by the Scientific Advisory Board during the pandemic, were provided by the Ministry of Health and delivered to those concerned at no cost.

The significant success of the Turkish healthcare system in universal health coverage has become very visible during the pandemic. Turkey pays regard to complete fairness in access to healthcare services and does not identify any disadvantaged groups. Turkey has shown a decisive attitude in providing cost-free comparable healthcare services to asylum seekers as well as Turkish citizens. The robustness of the healthcare system is not related to the investments in 
healthcare and, therefore, the abundance of opportunities, but related to the extent of availability to all.

\section{Healthcare Human Resources}

Qualified healthcare professionals are one of the strongest characteristics of the Turkish healthcare system in bringing the COVID-19 pandemic under control and preventing the devastating consequences that have occurred in some countries. Although the number of persons working in healthcare is low compared to international statistics, such as those of the European Union and the OECD, effective use of Turkey's well-trained and motivated healthcare workforce enables successful diagnosis, treatment and care services, contact tracing and surveillance processes.

It may be considered that an intensive workload due to the limited number of healthcare personnel before the pandemic played a role experience-wise in the success of undertaking the burden in this period. The quality of healthcare professionals and their sense of commitment to the profession are just as important as their number. During the pandemic, Turkey placed a particular emphasis on motivational policies as far as healthcare workers are concerned.

Turkey has over a million healthcare professionals with the addition of other healthcare workers and support personnel. In terms of human resources, the Turkish healthcare system has had the opportunity to provide necessary reinforcements to regions that needed additional personnel to combat the pandemic. In order to support its health worker capacity, Turkey employed 32,000 additional medical staff in April 2020, thus reinforcing its measures to fight the pandemic.

\section{Medicines and Medical Equipment}

During the COVID-19 outbreak, the need for ventilators in intensive care units in European countries and China prompted Turkey to speed up the manufacturing of ventilators at home. A prototype had previously been developed by a start-up firm, and the mass production of respiratory equipment was initiated with the cooperation of national firms under the coordination of the Ministry of Health. Turkey did not have any difficulty in terms of intensive care resources and even exported some of the domestically produced breathing apparati.

The most critical step in the fight against the COVID-19 pandemic is the development of vaccine and drugs. In this respect, Turkey has rapidly set to work concurrently with other countries. In this context, vaccine projects and chemical and biotechnological drug development projects are being carried out under the coordination of the Scientific and Technological Research Council of Turkey (TÜBİTAK). Some of the medicines that have been used in the treatment of the disease during the 


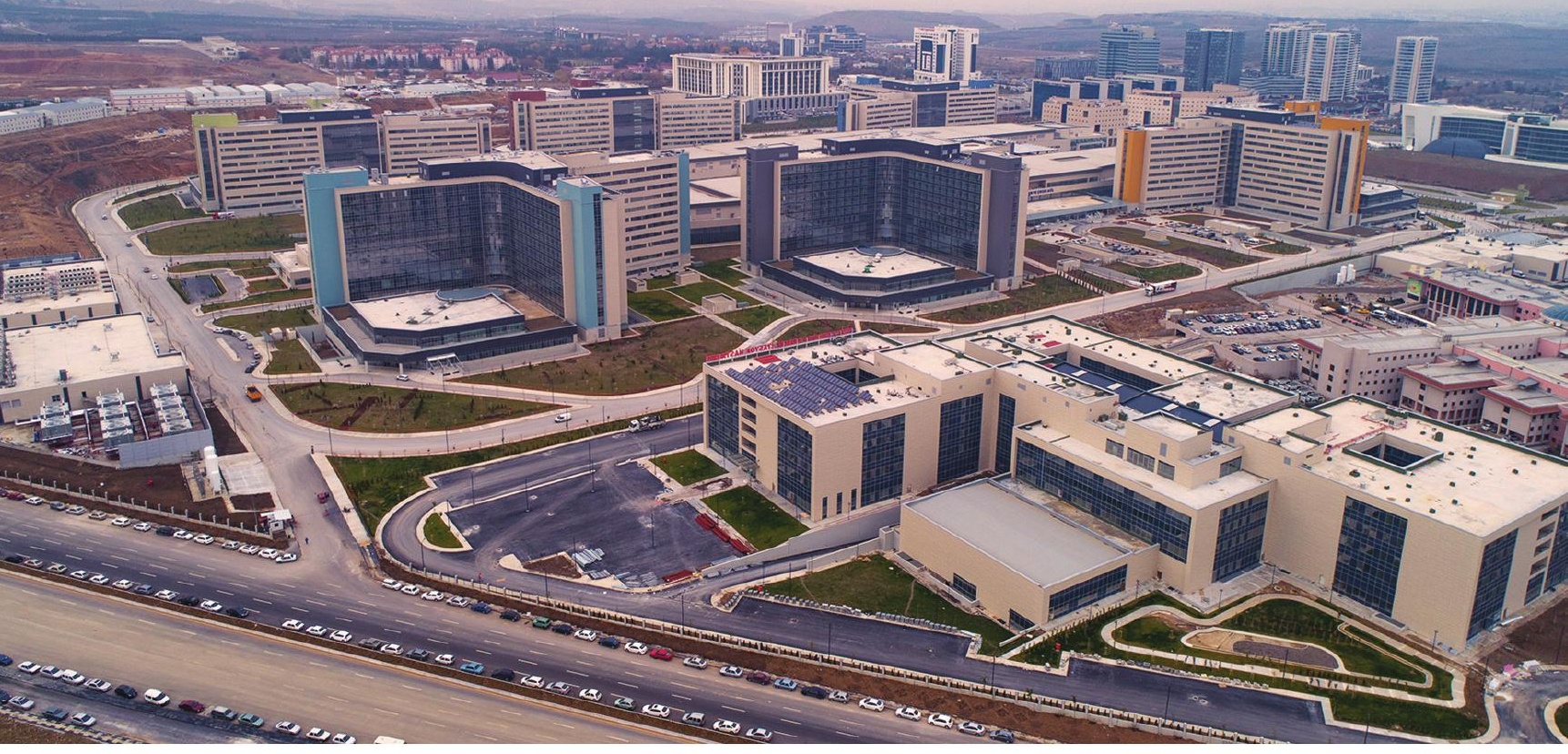

Ankara City

Hospital,

the biggest comprehensive health complex in Europe and the world's third biggest hospital, with over 3,700

bed capacity, was built in record time and opened in 2019.

Ankara Şehir Hastanesi pandemic and their raw materials are produced domestically.

The Vaccine Institute was established in 2018 under the Department of Health Institutes of Turkey (TÜSEB) and the Scientific Advisory Board of Vaccine affiliated with the Institute started the work. Moreover, the Department making calls for project cooperation in drug development, medical devices and biomaterials, diagnostic test kits and vaccine development declared support for clinical trials.

The COVID-19 pandemic has, once again, revealed the importance of domestic and national vaccine production. The period of the outbreak further demonstrates how essential the production of simple personal protective medical materials, such as face masks, disinfectants, gloves, and overalls is. Turkey is more fortunate than many countries in terms of these materials. Despite ephemeral setbacks in supplying protective materials, Turkey is a self-sufficient country in general.

One of the most important steps Turkey has taken in the fight against COVID-19 is the development and use of test kits produced locally. Ten domestic firms, having patented technologies in their portfolios, produce COVID-19 diagnostic test kits, some of which are exported.

\section{Providing Healthcare Services}

Turkey has succeeded in the management of the COVID-19 pandemic thanks to the tremendous, coordinated efforts of all healthcare units and personnel from the first level to the third in healthcare services. Family physicians and family healthcare professionals have contributed greatly to COVID-19-related contact tracing, diagnosis and follow-ups. As of June 2020, a total of 2,985 '112 emergency' aid stations, 5,437 ambulances, 17 helicopter ambulances 
and three air ambulances have been placed in service across Turkey. During the pandemic, air ambulances were frequently used, particularly for the transfer and repatriation of Turkish citizens living abroad.

As of March 2020, Turkey had a total of 1,524 hospitals, 245,422 registered beds $(41,593$ of which are intensive care beds) and 30,722 ventilators in all sectors. During the pandemic period, 794 hospitals were declared pandemic hospitals and 11,269 hospital beds were designated as isolation beds.

Secondary and tertiary health institutions were adequate and able to meet the needs of the population in the conduct of treatment processes. Increasing the number of single-patient rooms across the country with the policy of increasing the qualified patient beds has been recently achieved in Turkey. Thus, care protocols for patients have been designed and isolation precautions have been taken. With their qualified and single-patient rooms, scores of intensive-care beds and units equipped with state-of-the-art medical devices and materials, city hospitals, which were established via a public-private partnership model, have played a major role in the delivery of services -especially in big cities. All of the rooms in the city hospitals can be converted into intensive-care rooms. This is an unprecedented practice that will make a difference in the effective fight against COVID-19.

Thanks to the public health laboratories, some universities and a num-
When a coronavirus case is identified in any of Turkey's 81 provinces, the business and family information of the person who tested positive automatically enters the Ministry's system

ber of COVID-19 test centers that have been put into operation within TÜSEB, a test capacity of more than 50,000 tests per day has been achieved. We have not experienced any overload in our hospitals or intensive care units for the following reasons: (i) Applying high pressure oxygen therapy to patients with pulmonary involvement before intubation as advised in the treatment protocol prepared by the Scientific Advisory Board, (ii) starting drug treatment at an early stage, and (iii) having a strong infrastructure and talented medical staff.

In order to be prepared for a pandemic, the Turkish government had previously decided to increase its capacity and prepare İstanbul for all kinds of outbreaks, earthquakes, and disasters. To this end, during the coronavirus pandemic, two modern earthquake-resistant hospitals, one on the European side and the other on the Anatolian side of the city, were built by the government according to a horizontal architectural approach and opened in a very short time (45 days). The hospitals, each having 


\section{Our country has become} one of the countries that successfully carried out the management of the COVID-19 pandemic with a proactive, holistic, and dynamic approach under the leadership of our President, Recep Tayyip Erdoğan of infectious diseases. Filiation and contact tracing are of a great deal of importance in the control of the pandemic.

Teams of filiation and contact tracing personnel consisting of first-level healthcare workers, in particular, were formed to keep the COVID-19 pandemic under control in order to prevent its devastating consequences. Thanks to these protocols, isolation and quarantine measures could be taken rapidly. When a coronavirus case is identified in any of Turkey's 81 provinces, the business and family information of the person who tested positive automatically enters the Ministry's system. At this point, the teams of filiation and contact tracing consisting of at least three experts form a new network and get to work against the disease in every single new case of COVID-19. Since the beginning of the pandemic, a total of 6,200 teams have been formed and 985,403 contact tracing processes have been completed with 4.7 persons per case contact-traced.

\section{Family Medicine}

The healthcare system in Turkey has managed the COVID-19 pandemic effectively and efficiently thanks in part to strong primary healthcare services. Preventive measures and quarantine have made an important contribution to individuals' responsibility and consequently their literacy regarding healthcare. Every person is registered to a physician in the system of family medicine, which plays 
an important role in the follow-up of the identified contacts.

\section{Health Tourism}

Health tourism has gradually gained increasing importance in recent years and the need/demand for destinations that can offer health services with qualified personnel and equipment at a reasonable cost continues to increase. As a result of Turkey's successful fight against COVID-19 and its outreach to more than 80 countries, the recognition of Turkey's healthcare services has increased worldwide; its positive perception has resulted in a global public perception of these services as internationally qualified.

Equipped with all kinds of modern infrastructure and devices, and each having a 1,000-bed capacity, emergency hospitals were recently built in İstanbul's Sancaktepe and Yeşilköy neighborhoods. Both locations are in close proximity to international airports. These hospitals are designed to be used in the event of outbreaks, earthquakes or other disasters, and are expected to make significant contributions to health tourism in Turkey.

At the beginning of the normalization period, Turkey actively implemented the necessary measures and procedures for health tourism. Starting from airports and border gates, the procedures for the transportation of foreign patients and their companions to hospitals, their treatment and follow-ups were defined. Through these measures, Turkey intends to manage foreign patient traffic in a controlled and secure manner.

\section{Conclusion}

The characteristics of different countries and their varying practices in combatting the COVID-19 outbreak have led each country to experience the process differently and face different results. Healthcare systems in many countries have been clogged, infrastructures for healthcare delivery have been exposed as insufficient, risk groups have not been contained, mortality rates have become higher than expected, and decision-makers and healthcare professionals have faced ethical dilemmas regarding measures, treatment and service delivery.

However, Turkey has displayed and continues to display a successful performance in the fight against COVID-19. Our country has become one of the countries that successfully carried out the management of the COVID-19 pandemic with a proactive, holistic, and dynamic approach under the leadership of our President, Recep Tayyip Erdoğan.

The mortality rate of the COVID-19 pandemic in Turkey is among the lowest compared to many countries. Thanks to the steps taken by the healthcare sector and other sectors, Turkey has brought the rate of infection under control and has conducted diagnostic and treatment processes in an effective manner. 


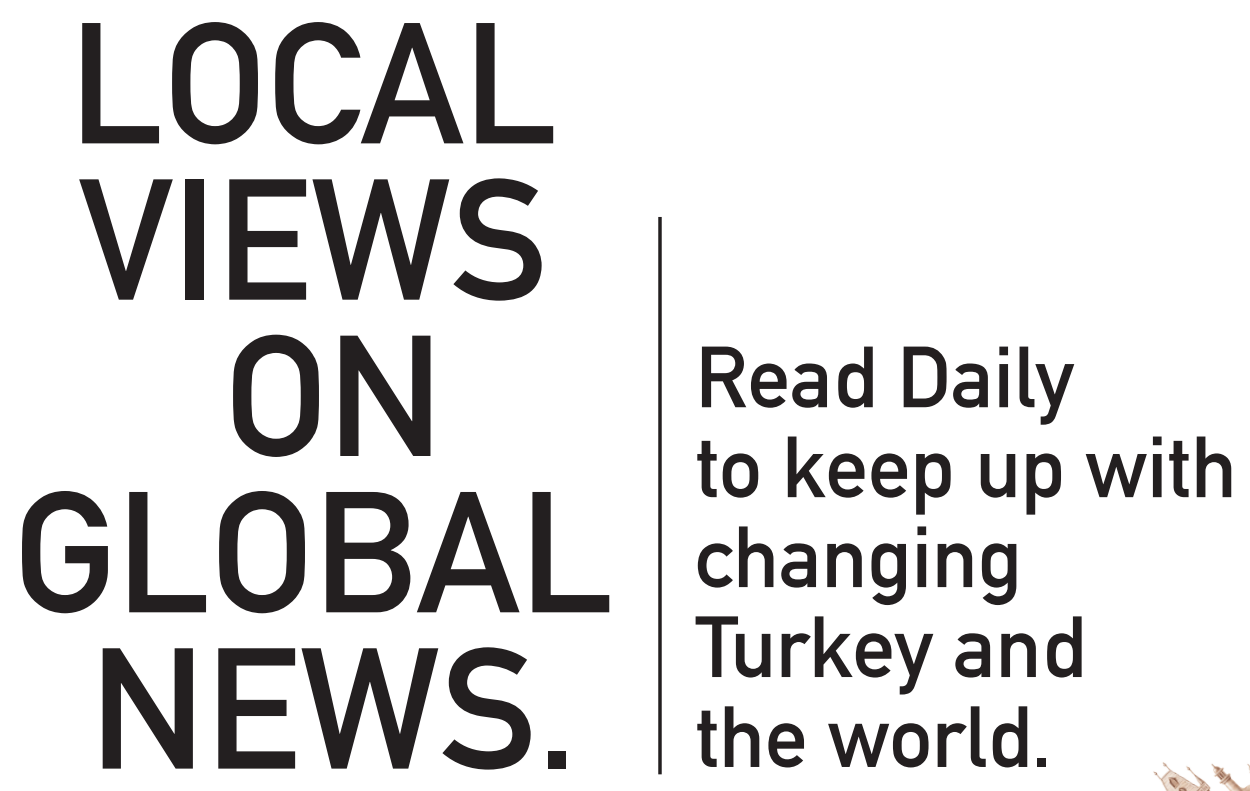

\section{DAILY 马AВAH}

